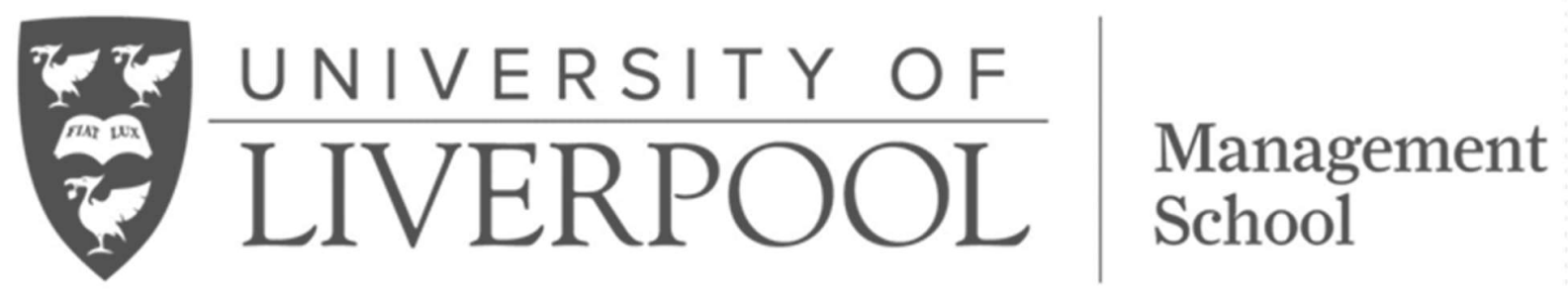

Working Paper in Economics

\# 202015

May 2020

\title{
Asymmetric Information in Menstrual Health and Implications for Sustainability: Insights from India
}

Supriya Garikipati 


\title{
Asymmetric Information in Menstrual Health and Implications for Sustainability: Insights from India
}

\author{
Supriya Garikipati ${ }^{1}$ \\ Management School, University of Liverpool
}

\begin{abstract}
This article explores how markets for menstrual products evolved and its implications for sustainability of menstrual hygiene management. The focus is on low-and-middle-income countries where $85 \%$ of girls and women of menstruating age live. I draw on a combination of secondary literature and focus group discussions with women from urban slums in India. My findings suggest that a tangled web of traditional taboos, markets and government policies have merged to create and endorse asymmetric information in menstrual health that has promoted the single product category of disposable pads. This has deeply influenced the beliefs and behavioural practices of menstruating women, which in turn have adverse implications for environmental eco-systems. It also seriously limits women's agency in the choice of menstrual product as awareness of alternatives is negligible. Analysing women's responses when they are offered information on other menstrual alternatives suggest that, as a policy tool, 'informed choice' has the potential to steer the menstrual health markets in a more sustainable direction.
\end{abstract}

\section{Evolution of Markets for Menstrual Hygiene Products}

For a large part of human history, menstruation was surrounded by traditional taboos that prevented people from openly discussing it. These taboos largely originated from religious beliefs that considered period blood as 'dirty blood', both 'impure' and 'unclean'. This was used to justify the restrictions placed on menstruating women across the millennia (Guterman, Mehta and Gibbs, 2007). Overtime these beliefs hardened into a widely accepted narrative that menstruation was 'shameful and embarrassing' and women must hide this bodily function from everyone around them.

This culture of shame and silence around menstruation meant that little happened in terms of innovations in menstrual materials until the turn of the $20^{\text {th }}$ century. This changed with the invention of cellucotton - a super-absorbent material used as medical bandaging during world war one (Museum of Menstruation and Women's Health, 1988). Nurses started to use it as sanitary pads, while some women gravitated towards using them as tampons. These ideas stuck and the era of disposable menstrual products began. As more women joined the workforce, demand for disposables started to increase in the US and in the UK and by the end of world war two, this change in habit was fully established.

Marketing campaigns furthered this demand by leaning heavily into the idea that using disposables freed women from the 'oppressive old ways', making them 'modern and efficient' (Borunda, 2019). The profit incentives were considerable as disposables locked women into a cycle of monthly purchases that lasted for several decades. Driven by profits, commodification of comfort and convenience created an entirely new market segment for female hygiene products.

\footnotetext{
${ }^{1}$ Research assistance by Tanya Malik and Linda Mason are gratefully acknowledged. The usual disclaimer applies.
} 
Technological advances in flexible plastics over 1960s and 70s saw disposables become more leak-proof and user friendly as plastic back-sheets and applicators were introduced into their designs. Another notable innovation was the introduction of non-woven absorbents in the making of the core of pads and tampons. As these products became more efficient in 'hiding' menstrual blood and woman's 'shame', their appeal and ubiquity increased.

Most of the initial market for disposables was limited to the west, but in the 1980s some of the larger companies, recognised the vast potential for market expansion into low-and-middle income countries (LMICs). Once again, they created the aspiration for comfort and convenience, but this time for middle-class women in LMICs. With changing lifestyles, these aspirations became a necessity for these women. Given that cultural taboos had left a veritable vacuum of information around menstruation in these countries, it was easy for the companies to position sanitary pads as the only means of hygienically managing periods.

Their efforts received a considerable boost in early 1990s when global concerns around the menstrual health of poor girls and women in LMICs started to take hold and public health initiatives began to endorse the idea that disposable pads are the only hygienic means to manage menstruation. However, the ubiquity of sanitary pads coupled with the lack of recourse for handling increasing volumes of menstrual waste in these countries has led to a growing recognition of their negative impact on the environmental eco-system (Peberdy, et al., 2019).

Disposable pads generate an estimated global waste of around 480 billion soiled pads annually (van Eijk et al., 2019). Managing menstrual waste is a huge challenge in LMICs, especially in face of unprecedented urbanisation (WHO, 2010). Recent figures suggest that over 229 million girls and women of menstruating age in LMICs live in urban slums (UN, 2019) and manage their menses in squalid conditions that are typically lacking in wet waste management systems (Garikipati and Boudot, 2017). Most used pads would thus end up either in landfills or in the oceans where the plastic and other non-compostable material in these products takes hundreds of years to decompose (Thought Co., 2013).

Worries over the potential global menstrual waste burden and concerns over the sustainability of these products and increasing awareness of limitations to urban waste management in LMICs have seen the global MHM discourse move to the dual recognition that whilst good menstrual hygiene is central to the health and wellbeing of women, there is an equal and urgent need to reduce the environmental impact of menstrual waste and promote more sustainable materials.

Over the last few years, there has been a marked shift towards the study of more sustainable materials like reusable cloth-pads and menstrual cups (Peberdy et al., 2019). For instance, a study from Uganda reports that schoolgirls using reusable pads report less difficulty and disgust with cleaning and changing absorbents and increased absorbent reliability (Hennegan et al., 2016). A study from western Kenya reports that the provision of disposable pads or menstrual cups reduces the exposure to sexual and reproductive harms among schoolgirls with a $\sim 50 \%$ lower prevalence of sexually transmitted infections compared with usual practice (BenshaulTolonen et al., 2019).

Despite all this, there is as yet a lack of consistent effort by state and civic society stakeholders in LMICs to include more sustainable alternatives to disposable pads in their MHM policies and programs. At least partly, this is because we lack an understanding of how promotion of 
disposable sanitary pads has influenced menstrual beliefs and practices among women. We also lack an understanding of women's awareness, attitudes and preferences for environmentally sustainable alternatives.

Home to over $20 \%$ of the world's menstruating girls and women, India represents an important case to examine these issues (estimated from Census of India, 2011). Like in many LMICs, disposable pads have dominated the menstrual health discourse in India where markets, government policies, consumer voices and media conversations condone and reinforce the narrative that menstrual health and hygiene is equivalent to having access to sanitary napkins (Muralidharan, Patil and Patnaik, 2015).

In this paper I use focus group discussions with women from urban slums in India to understand how the experience of equating menstrual health to a single product category influences beliefs and behavioural practices among menstruating girls and women. I examine how, confronted with limited availability and affordability, women weave in the use of traditional materials like cloth with the use of disposable pads. I also explore the influence of other factors in their lives and their environment that impact their menstrual behaviour and practice and make these hygienic or not. I also examine practices surrounding disposal of used pads and ask if women consider the environmental impact of pads when disposing them. What about awareness of other environmentally friendly alternatives to disposable pads? Where there is a lack of awareness of these alternatives, how do women react when they are given unbiased information about alternatives? Does informed choice have the potential to be a useful policy tool to steer the menstrual hygiene markets on a more sustainable path?

The insights from these conversations help understand how the current menstrual product market influence menstrual practices and their implications for sustainability. They also open a window to potential answers that can lead to more sustainable solutions - both for the women consumers and for the environmental eco-systems.

\section{Menstrual Hygiene Markets and Policy in India}

Over $85 \%$ of the world's two billion girls and women of menstruating age live in LMICs (World Population datasheet, 2017). The awareness that their menstrual health intersects with several Sustainable Development Goals led to its inclusion in the mandate of WHO, UNICEF, UNFPA, UNDP, and other agencies. The effects of poor menstrual health on girls and women was also identified as a neglected component of public health systems in LMICs and global advocacy around this issue has been growing over the last 20 years (Sommer, et al., 2013).

The term 'Menstrual Hygiene Management' (MHM) was coined to describe what was necessary for girls and women in LMICs to maintain good menstrual health. MHM implies that adolescent girls and women have the knowledge and awareness to manage their menses, they have access to clean menstrual material to absorb and collect blood, that can be changed in privacy as often as necessary, using soap and water for washing as required and be able to access facilities to dispose used menstrual materials (UNICEF, 2014). The goal of MHM policy must then be to ensure healthy practices throughout this menstrual value chain via the delivery of interventions. Internationally, however, it is noted that one component of MHM provision or access to sanitary materials - has been favoured in the public policy arena, and this has largely been managed via the distribution of free or discounted single-use sanitary 
pads to schools mainly due to the tangible nature of such interventions (African Coalition for MHM, 2019). Pads were preferred over tampons because taboos against vaginal insertion prevail in many parts of the world.

India is a deeply patriarchal country where traditional taboos related to menstruation are still widely prevalent (van Eijk, et al., 2016; Sivakami, et al., 2018). Conversations around menstruation have been mired in a culture of silence, resulting in an absolute black hole of information around the topic, especially for menstruating women who could not speak to anyone about their experiences or needs (Mahajan, 2019). In was in this context that a few decades ago, some large pad manufacturers infiltrated this space to position disposable sanitary pads as a solution. With 400 million girls and women of menstruating age living here, India represented a sizeable market.

But for a long time, pads were still only accessible to a small segment of Indian women. In 2011, the NGO Plan India reported that just $12 \%$ of Indian women could access sanitary pads (Plan India Annual Report, 2012). This raised concerns around the challenges faced by women using traditional solutions in maintaining their menstrual hygiene and personal dignity. While cloth is a hygienic menstrual solution (Torondel, et al., 2018), it requires adequate washing and drying, which is difficult to achieve in a country where taboos about menstrual blood are prevalent (Baker et al, 2017).

These concerns led the government of India to design national guidelines in 2015 and a string of strategies for the adoption of good hygiene (National MHM Guidelines, 2015). The dominant paradigm in these strategies is the free or discounted distribution of disposable sanitary pads. While guidelines in India acknowledge and guide on how to maintain hygiene using cloths, government MHM initiatives favored disposable pads over traditional items. This may reflect the concern that cloths are inadequately cleaned, leading to infection, but it also shows a readiness to opt for a quick and easy solution where single use pads are distributed as the main MHM-related activity, since infrastructural changes on WASH and other supplies are difficult logistically.

Most prominent among the government's MHM initiatives is the national flagship programme, Rashtriya Kishor Swasthya Kayakram, operating through the National Rural Health Mission, which promotes girls' access to sanitary pads (Muralidharan, Patil and Patnaik, 2015). The most recent example of this is the allocation of Rs. 100 crores by the Government of Andhra Pradesh to the distribution of sanitary pads to 20 lakh adolescent girls (Jayachandran, Jain and Dhar, 2018). Distribution of pads has been supplemented by the extensive push on small-scale manufacturing units for sanitary pads which offers the added promotion of livelihoods for women (Venema, 2014; Sommer, et al., 2012).

By focusing on comfort and convenience, marketing campaigns have created a huge aspiration demand for pads. Influences by media have reinforced this narrative in popular appeal, the most well-known example of which is the resounding success of the Bollywood movie 'Pad Man' (based on the story of a social entrepreneur who invented a machine for the manufacturing of cheaper disposable pads, Waheed, 2018). The lead actor of the movie, later became the celebrity face of government media campaign to popularise the use of pads.

Markets were not far behind. Large manufacturers, sensing the potential in the Indian market - especially among the low-income segments - introduced a range of product variants and promoted brand and aspirational awareness through multi-media networks. By now there are 
variants in the market that range from Rs.2 per pad to Rs12 (National MHM Consultation, 2017). Low-price products are also available in small pack sizes of six for better affordability. Companies have access to the medical retail network in India and have the potential to leverage the full extent of their consumer goods retail network.

Given government promotion and aspirational marketing by the private sector, menstrual hygiene in India has become akin to access to sanitary pads. Cheap commercial variants, government efforts and private philanthropy combined to cause a rapid surge in demand for sanitary pads. In less than five years, the National Family Health Survey (NFHS) in 2015-16 reported pad users to have quintupled to $58 \%$, with rural users at $48 \%$ and urban users at $78 \%$ (NFHS, 2016).

Using these estimates we calculate that there are 121 million pad users across India. If we assume women use 8 pads per cycle on average (allowing for differential usage in urban and rural areas) this gives us 1.02 billion pads used every month. Using the PATH waste-loading model which gives us the dry mass of each disposable pad as $9.2 \mathrm{gm}$ (Wilmouth, et al., 2013), we can estimate India's current menstrual waste as 9400 tonnes per month or 112,800 tonnes annually. This represents a significant disposal burden, especially for cities where wet waste systems are already overwhelmed and open sewers clogged with soiled pads is now a common sight.

What about sustainable alternatives to pads? While there is no concerted public effort towards informing women about sustainable alternatives to disposable pads, we found several small initiatives. Particularly noteworthy is the menstrual cup initiative by the government of Kerala (The Logical Indian, 2019). Launched in 2019, the Thinkal project distributed 5000 menstrual cups free of charge to women from the municipality of Alappuzha. The idea emerged out of the devastation caused by floods in 2018, where women in the relief camps faced a massive problem with the disposal of their sanitary pads. Other noteworthy initiatives are Uger's (Jatan Sansthan, 2001) and EcoFemme's (EcoFemme, 2010) versions of reusable cloth pads, Safepad's (RealRelief, 2016) reusable pads with anti-microbial coating and Anandi's (Aakar Innovations, 2018) compostable pad which needs deep burial for composting. Without the backing of government policy and funding, however, these efforts remain small and sporadic and have little overall impact on knowledge and consumer behaviour.

\section{Study, Data and Methodology}

In the above context, this study examines the menstrual beliefs, behavioural practices and knowledge among women living in slums across the city of Hyderabad, capital of the state of Telangana in south India. Data comes from a larger study that examines changes in women's preference for menstrual materials when exposed to various menstrual products (Garikipati, Docherty and Phillips-Howard, 2019). Here I examine the qualitative data that was gathered via focus group discussions (FGDs) with 70 women from these slums. Two sets of FGDs were planned. The first set focused on 'Practices, Awareness and Beliefs' and the second set focused on 'Attitudes and Acceptance of Sustainable MHM Alternatives'. Five FGDs were carried out in each set, resulting in a total of 10 FGDs.

The study was funded by a GCRF award 2016/17 (Ref No. 141131). The project received ethics approval from Safa in Hyderabad, India (Ref: Safa0317R) and University of Liverpool in Liverpool, UK (Ref: RETH000734). Study period was between July 2017 to May 2018. 
To ensure a collaborative research process, research was carried out in partnership with four grassroot social organisations: Safa, KGNMT (Kasturba Gandhi National Memorial Trust) Karthavya foundation and Streedhan. Each of these organisations works with vulnerable women in some capacity, either to raise awareness of social issues or to enhance their livelihoods and empower them economically. In consultation with partner organisations, ten slums were selected to achieve a mix of slum size, access to amenities and other considerations like safety of researchers and accessibility. In order to get a more representative measure of MHM practices across slum types, we also included both notified and un-notified slums - the former are officially registered with the municipality and are meant to have better WASH (Water, Sanitation and Hygiene) provisions in terms of garbage collection, access to water and public toilets, whereas the latter are unregistered and hence have no claims to the city's WASH provisions. Although they may be served by local NGOs and private philanthropists. Using a mix of municipal records, records maintained by partner organisations and women community-leaders, a list of households in the slums was prepared.

We then randomly selected up to twenty women from each slum and invited them to participate in a focus group discussion around the issues concerning women's health. A time and place were decided in advance with NGO partners and community leaders. Typically, this was either in the NGO's office or in another neutral private area like a community hall. All FGDs were recorded with prior verbal consent from women. We did not take written consent as literacy levels were highly variable, especially among older women and taking written consent may be discomforting to those unable to read and write. Recorded FGDs were then sent for translation to English and transcribed. To support triangulation of results, we also carried out two interviews with sanitation workers who are responsible for sifting waste in the notified slums and interviewed local stockist of menstrual products.

All transcripts were stored onto Nvivo (Version 11, QSR International) to facilitate sorting and coding of textual data. The first step involved reading and re-reading the transcripts to establish familiarity with the data and for contextualisation of the women's narratives. This was carried out by a researcher who was not involved in the preparatory stages of the research nor collection of the data, thus had no preconceptions of what would emerge from the findings thereby facilitating an unbiased analysis using an inductive process. Familiarisation of the data enabled an initial coding framework to be devised. Each transcript was then coded using the framework in a step by step manner with new codes added to the framework where appropriate. Any redundant codes were removed. The resulting framework was sorted into a logical order whereby overlapping, similar or related codes were organised within a series of sub-themes. The same process was applied to the sub-themes resulting into a smaller number of key themes. The data within each theme were woven into a narrative with key quotes included to illustrate the point and provide context for the reader. To ensure objectivity, the author familiarised herself with data from the transcripts before checking to ensure that the written narrative epitomised the findings from the FGDs, and that the quotes used were appropriate in representing the nuances and meanings of the key themes.

\section{Findings and Discussion}

In this section, I begin by using information from the FGDs to understand the types of menstrual materials used by women participants and their menstrual practices. The focus is on exploring the influence of traditional beliefs and other environmental factors (like access 
to limited product types and living conditions) that may render menstrual behaviour hygienic or unhygienic.

\section{Menstrual materials and user preferences}

Use of menstrual protection among the women who participated in FGDs was split between cloth and disposable pads, although on balance a higher proportion claimed to use pads. This reiterates the NFHS 4 findings for all India. Indeed, all members of two younger FGDs (participants aged 18-20 and 20-30 years), stated they used disposable pads.

Some women noted that although they used cloth in their village, they now prefer pads because using cloth is "not modern', "we live in the city so we use pads', 'cloth is old style' (FGD1.4). Others talked about having used cloth when they were younger then changing to pads as they became more accessible. In fact disposable pads were the only menstrual product that local shops stocked. Participants of the remaining FGDs comprised a mix of cloth and pad users, including some who used cloth when at home but used a pad at work. There also appeared to be some generational differences with women mentioning that their mothers and mothers-in-law used cloth but their own daughters used pads. Overall, these conversations suggested that as pads become more affordable and as older users leave the market, using pads is likely to become the norm.

Users of pads purchased these from local chemist or grocery shop and liked them because they were comfortable and convenient. Local stockist of menstrual products only stocked disposable pads, typically just a couple of the top brands. Pads also had the advantage of being thick and having wings thereby not causing stains through leakage 'its good, no stains so tension is not there. This is the best thing' (FGD1.2) Whilst her companion added '... now use and throw, no tension' (FGD1.2). Some women spoke about variation in quality of pads and agreed that only cotton pads were good. Indeed, it was mentioned in one FGD that other materials in pads has caused rashes.

The advocates of cloth liked them because they are free or cheap, and easily obtainable. Most utilised materials they had at home, in particular saree material as it was cheap and plentiful. , Women reported using cloth as a matter of habit, 'we didn't have pads before, we started with cloth and we got used to it' (FGD1.2) A couple of women mentioned comfort as a reason 'cloth is of cotton material and its' soft and thin' (FGD1.3). However, other women had nothing positive to say about using cloth for menstrual protection and. When asked what is good about using cloth, one vehement response was 'Nothing! There is nothing good in it' (FGD1.4). Another FGD had a similar discussion with participants not liking cloth because it causes fungal infection, it moves around, and chafes the skin 'it peels my skin....yes if you walk for a while it peels the skin' (FGD1.5) Many women, both cloth and pad users, consider cloth to be unhygienic. The word 'galeez' - which is the Hindi word for 'dirty' or 'unhygienic' was used by several participants to describe menstrual cloth. The aspiration to switch to pad among most cloth users was high.

\section{Menstrual practices: the influences of traditional beliefs and environment}

Exploring practices by cloth users helps us understand how traditional taboos and beliefs surrounding period blood and other environmental factors impacted behavioural practices and how these in turn could be perpetuating the belief that cloth is unhygienic.

Many of the women did not have access to private washing facilities which also limited their ability to wash cloth properly. Some cloth users threw away the material each time it required 
changing as they did not like to touch cloth soiled with period blood. Others described washing their cloth with soap which they kept separate from other household soaps, and similarly they used a brush to scrub the material that was only used for cleaning menstrual cloth. Some excerpts from descriptions of how cloth was washed show how traditional beliefs about period blood guided menstrual practices:

'We first squeeze the blood out with our feet, then wash it with soap' (FGD2.5) 'the soap is separate we cannot wash other clothes with that'(FGD1.4), 'yes, even the brush is separate for washing that cloth' (FGD1.2; FGD2.5).

Traditional taboos also influenced how women dried their menstrual cloth. Women also choose not to dry cloth under open sunlight for the humiliation of being seen by male members of the family and outsiders. Drying cloth in the open was considered 'shameful' and 'not the right thing to do' (FGD1.4). Other excerpts that suggested this,

'People shouldn't see it' (FGD1.2)

'Kids or elders shouldn't see it' (FGD2.5),

'It is not good (to dry it in the open)' (FGD1.2; FGD1.4)

Women tend to dry their menstrual cloth indoors, concealed in closets and hidden underneath other material to keep it hidden;

'When we use cloth, before bathing we wash it clean with soap and all, then we dry it differently and we put a different cloth on it. No one notices. And once it is dry we fold it and keep and then that can be used next time' (FGD1.3)

There was some discussion in a couple of FGDs that this 'need to hide' was partly a consequence of slum living and that it was easier in a village setting where materials could be dried outside as 'No one will notice, here most of them could see' (FGD1.2)

Such washing and drying practices render the use of cloth unhygienic (also see Baker, et al., 2017). It is also likely that the myths and taboos that limit women's ability to wash and dry cloth in a hygienic way contribute to the belief that cloth is inferior to pad.

The general culture of silence and shame around periods meant that women did not feel comfortable seeking information from better informed individuals (health workers, teachers) and ended up believing what they are told by women in the family and friends. This resulted in blurring of boundaries between myths and reality and what was repeated over and over again became a 'period fact'. Although it was common for women to have had some schooling and for younger girls to have studied in college with some attending university, formal education, we found, made little difference to beliefs about menstrual products. A college student who participated in our study told us that "cloth was bad because my aunt's friend became infertile because of it" (FGD2.1).

We also found unhygienic practices among the pad users - like using the same pad for the whole day or to use it on two consecutive days if the flow was light. Commenting on such practices, participants in one FGD stated '...some women use pad for more than six hours then that too causes infection' (FGD1.5). This suggests that lack of information on correct use of pads may mean that its hygienic use cannot be taken for granted in all circumstances.

\section{Disposal practices}

What happens to used menstrual materials? Do women consider the environmental impact of pads when disposing them? 
The vast majority of the women in our study threw their pads out with routine waste and it was convenience rather than environment that was foremost on their minds when disposing used pads. It is also common to see soiled pads floating in open streams and gutters next to dwellings. Women told us about discarding used pads in the waterways close to their homes as it was the most convenient way of disposing it.

Correct disposal of pads according to our participants tended to be by wrapping of the pad in the packaging that came with it or a plastic bag and discarding in the dustbin. Women spoke about what they perceived as wrong or bad practice 'some women just throw it open like that everywhere in the bathroom' (FGD1.4). Littering of public spaces by discarded sanitary pads was acknowledged and a talking point in 4 of the FGDs with many comments along the same lines as 'and people don't care about disposing it right, they throw it anywhere, even in the bathroom it's lying open like that' (FGD2.5). 'Even if it is thrown in the river it stays with you, yeah and it comes back when it overflows and is lying all around. Then even children ask what is this? Then what are we supposed to say' (FGD2.5).

Like the drying of menstrual cloth, women were adamant that sanitary pad disposal should be kept secret, particularly from men. Yet they acknowledged that this was not possible with the current way that the pads were discarded or the garbage was collected.

'Main problem is, it's lying everywhere, even men end up seeing it and it is so embarrassing. They may not know what it is but we women know that it is our thing' (FGD2.5).

'There is only one way in or out of this area, right? So, we all have to walk there and we can see it everywhere, even boys see it' (FGD2.5).

That pads were lying on the ground visible to all seemed to be the only concern that women spoke of freely in relation to environmental pollution. Indeed, some participants themselves admitted to poor disposal methods including burial of pads in the wet mud alongside the river, whilst others tossed them directly into the river. 'See, we don't throw it in the dustbin, we just throw it in the river'; 'yes wrap in a plastic bag and then throw' (FGD2.9).

There was general awareness that garbage collectors sometimes just dispersed it further around the slum as it fell out of the bins, whilst the river overflows with pads and other materials at times, as illustrated by the following quotes. Participants in their late twenties told us: 'I'll tell you I have seen that many people throw it in the dustbin like that and the garbage man takes it and it falls. He doesn't pick and it is seen lying in the morning just like that and it looks galeez' (FGD2.5)

Much of the wet waste sifting in India is done by sanitation workers manually. We spoke to a couple of sanitation workers involved in this work. Sharing some of their experiences with sifting used sanitary pads from other waste, they told us:

'When I handle this mess, I feel my life is cursed.' (SWI)

'Even though I am a woman, I curse woman's birth when I have to do this work.' (SW2).

The plight of the sanitation workers is pitiable indeed. But there is unlikely to be an easy solution to this issue - especially given the limited wet waste infrastructure in India's heavily populous cities. Any model of environmentally-friendly waste disposal is likely to incur significant costs. Are users willing to share some of the costs of any such initiative? 
When questioned whether they would be willing to pay for ethical disposal of menstrual materials there was general agreement across all but one group, where only a minority of the women admitted that they would be willing to pay for such a service. Those against it cited the convenience and ease of just throwing discarded pads in the river, particularly as other women would still continue to do this 'there's still so many people they will throw everywhere' (FGD2.6). For those women in favour however, there remained questions over whether it was worth the price, as well as requiring the need for discretion, an issue raised in two of the discussion groups. Referring to cost, a few women in one group suggested 300500 rupees per month but other group members disagreed and set a price of 150-200r which seemed to be the consensus across all but one of the groups. Women in this group proffered that they would only pay 50-100r per month. To put things in perspective, the average cost of a branded pack of six pads across two local shops is 40r. The same women also had concerns whether the price would be for the whole household noting there are many women in a house, and also whether men would be the disposal collectors as they felt it was not good for men to collect used pads. Lastly, these same women also considered that paying for disposal would be worthwhile if it saved germs from spreading as illustrated by the following excerpt: 'if no one will see it outside' (FGD 2.5 and women in every FGD1 expressed similar opinion) 'there won't be impurity outside' (FGD1.2)

'Yes, no impurity around' (FGD1.5)

These excerpts suggest that user-paid disposal models may be possible and this area of work may benefit from experimental trials.

\section{Knowledge of alternative menstrual materials}

The women who spoke to us during the fieldwork got their information on menstrual products from TV adverts and billboards. All of the women appeared to be aware of two types of menstrual management materials: traditional use of cloth and disposable sanitary pads, but very little was known about other menstrual products. None of the women had heard of commercially made reusable cloth pads and very few had heard of other alternatives such as menstrual cups. One participant in the young FGD2.1 (aged 18-20) mentioned that she had heard of tampons, as well as the menstrual cup and only one other woman, (a 20year-old) reported having heard of a tampon. Those aware of these products had heard about them through social media, mostly 'YouTube'. Participants in the other FGDs denied being aware of other alternatives when initially asked by the moderator, although some later showed some awareness of re-usable pads and the menstrual cup when the moderator spoke of these products. Majority of the women though lacked awareness of them.

\section{Informing women's choice of menstrual products}

Asymmetric information of menstrual products clearly limits women's agency in the choice of menstrual products. In this context it is relevant to ask what might happen if women had the correct information on all menstrual alternatives. This is the idea of 'informed choice' in menstrual health when women have complete and unbiased information on all menstrual products, including availability, lifecycle cost, hygienic use and implications for waste. Robust testing of this idea would need a full trial, but even recording women's responses and discussion to the moderator's question of whether they would try other menstrual materials that were kinder to the environment were insightful. Participants in each of the FGDs showed interest and appeared open to considering a change in their menstrual hygiene practices, although few had ever considered the environment previously.

Willingness to consider other methods of menstrual protection was also illustrated by the keen interest women showed through the many questions they asked about menstrual cups in 
particular, as well as reusable pad cloths. Health, comfort, cost and availability appeared in these questions and were generally more important to individuals than protecting of their environment. Some excerpt arising from all FGD in set 2:

'Yes, if it is comfortable then why not?' (FGD2.4).

'Yes, the same, if we come to know where we get it and it its good then we will surely buy, right?' (both FGD2.1; FGD2.2)

'if it is in budget then we feel good to use it, if it is out of budget then we feel that both are serving same purpose then why shift' (FGD2.5).

Other questions included,

'is there any problem'? (FGD2.2)

'Won't it hurt?' (FGD2.2)

'any rash and all?' (FGD2.5)

'...if we put inside does it cause infection?' (FGD2.5)

'... and blood won't leak?' (all FGD2.3)

'is there any problem while sitting or what if we lie down then?'(FGD2.5)

'It is good I heard, but while sleeping or anything how will it not move?' (FGD2.1 and FGD2.3)

Overall women wanted to know about any side-effects, the level of comfort and whether there was any pain, how to insert and remove and also the cost. Following provision of more detailed information from the moderator many women across the FGD's spoke of their willingness to try the menstrual cup

'I will try for sure' (FGD2.5)

'this will be good I feel' (FGD2.3; FGD2.5)

Reasons women gave for wanting to try included that it had no side-effects, did not stain or leak, was long-lasting with 'no need to change it time to time in a day' (FGD2.4). As one participant stated: 'If we buy this then it's easy and if we keep buying pads then it will cost much, if like we need 3-4 pads in a month then we spend right, it is better we spend once and it is easy for us. There won't be pain, no stain, tension, right?' (FGD2.3)

Two participants also mentioned advantages of not having to dispose of it, or being seen doing so. Comments included 'we feel embarrassed if anyone sees it they will come to know when we throw pads, but if there is menstrual cup then its good, we can clean inside only and keep it....actually dustbin is placed outside and we need to watch if anyone is coming so that will no longer be an issue, so in the bathroom itself we can wash it, then it's safe and good' (FGD2.1)

Fewer questions were asked about the re-useable cloth pad, and only in one FGD despite little awareness of them generally. These included how long it can be used for, whether it can be rewashed it in the bathroom and whether it would cause infection. The same issues were raised though in relation to whether the women might use in future - cost, comfort and health. Despite women's reluctance to wash traditional pieces of cloth, there was some interest in trying the re-usable pad.

'We will try for sure' (FGD2.5)

'what is the harm in trying? (FGD2.2)

'If we like then ok' (FGD2.2 and all agree) 
On balance however, there appeared to be more women preferring to try the menstrual cup rather than the re-usable pad although some women did prefer the latter as illustrated by the following quotes.

'I will try cloth pad because I'm scared - I have never seen the cup, what if it goes inside me and gets lost?' (FGD2.5)

'I feel the cup system that people will not understand, however the cloth pad, many people can try' (both FGD2.5; FGD2.6)

However, one method that would not be workable in the slum environment according to our participants, were compostable pads that required burying. Across all FGDs there was consensus that there was no room in the slums to dig a hole and bury any material.

Compounding this were the close living quarters to others whereby people would either know what was happening or would be curious to know. The following were typical excerpts illustrating their thoughts on this.

'no no, but I have a question, see we all have no place here, now we have managed to mostly get cemented houses finally and whatever houses we have is enough now. we can still deal with throwing it in the garbage like this but who will go and dig a hole, we need place for that right?' (FGD2.4 and all agreeing)

'but there are no such places, we will have to search them, where will you dig?'”(FGD2.3) 'and then we have to carry it, don't know where are such places, then find them, then dig it then bury. It's like people might say yes but none will do it' (FGD2.3 and FGD2.4)

\section{Concluding Remarks}

The menstrual hygiene landscape in India evolved within the bounds of asymmetric information that was the direct consequence of the traditional taboos related to menstruation and hence the inability of all stakeholders to talk about it openly. This situation was exploited by companies to create an imbalanced market in favour of disposable pads. Lacking in correct and comprehensive information, both about the adverse impacts of pads in terms of high life-cycle costs and environmental burden and about alternative products, policy makers and consumer voices endorsed disposable sanitary pads as the only hygienic method to manage periods. Rising aspirations to comfort and convenience have helped sustain the narrative that pads as the best way to manage menses.

Where some women do continue to use traditional cloth, cultural taboos around menstrual blood and shared wash facilities severely limits their ability to properly wash and dry cloth. This may increase the probability of rashes and infection, further contributing to the belief that cloth is unhygienic. Although women have not hitherto considered the impact on their environments in disposing pads, they are open to the idea of paying for sustainable ways of disposal. Even the minimum payment amounts suggested by women for such services are more than the average cost of a pack of pads. This indicates that experimenting with pay-asyou-go models for ethical disposal of menstrual pads is certainly possible, but it may be argued that these efforts must be subsidised by pad manufacturers in the first instance.

The menstrual hygiene landscape, both globally and in India, has evolved tremendously in the last few years, with a variety of sustainable alternatives to pads now potentially available to women. We see a number of such initiatives by smaller manufacturers in India, including the Kerala government's experiment with menstrual cups. While information about these products is generally lacking among women, it is clear from our discussions with them that 
there is curiosity to find out more about alternative products and also willingness to change behaviour. It then seems that the prevalent informational asymmetry around menstrual health and hygiene that places disposable pads on a pedestal has failed to ensure women's agency in their choice of menstrual products. Our findings suggest that if women had complete and unbiased information about alternatives then it is likely that some would choose products that are more sustainable in terms of costs to themselves as well as to the environmental ecosystems. As a policy tool, informed choice may have the potential to steer menstrual hygiene markets in a sustainable direction. It is time that producers, governments and consumer voices get behind it.

\section{References}

African Coalition for MHM, 2019. First East and Southern African Regional Symposium on Menstrual Health Management. https://acmhm.org/1052-2/<Accessed 9.16.2019>.

Baker, K. K., Padhi, B., Torondel, B., Das, P., Dutta, A., Sahoo, K.C., Das, B., Dreibelbis, R., Caruso, B., Freeman, M.C., Sager, L., Panigrahi, P., 2017. 'From menarche to menopause: A population-based assessment of water, sanitation, and hygiene risk factors for reproductive tract infection symptoms over life stages in rural girls and women in India'. PLoS ONE. 12(12): 0188234.

Batterman, S., 2004. 'Findings on an Assessment of Small-scale Incinerators for Health-care Waste'. World Health Organisation, Geneva.

Benshaul-Tolonen, A., Zulaika, G., Nyothach, E., Oduor, C., Alexander, K.T., Mason, L., Laserson, K.F., Phillips-Howard, P.A., 2019. Pupil absenteeism, measurement, and menstruation: evidence from Western Kenya. CDEP-CGEG Working Paper Series No. 74. Center for Development Economics and Policy.

Borunda, A. 2019. 'How tampons and pads became so unsustainable', National Geographic. https://www.nationalgeographic.com/environment/2019/09/how-tampons-pads-became-unsustainable-storyof-plastic/ <Accessed on 16 January, 2020>

Census Data of India, 2011. http://censusindia.gov.in/2011-Common/CensusData2011.html $<$ Accessed on 22 April, 2020>

EcoFemme, 2010. https://ecofemme.org <Accessed on 22 April, 2020>

Garg, S., Sharma, N., Sahay, R., 2001. 'Socio-cultural aspects of menstruation in an urban slum in Delhi, India'. National Centre for Biotechnology Information, Reproduction Health Matters. 9(17): 16-25.

Garikipati, S., Boudot, C., 2017. 'To Pad or Not to Pad: Towards Better Sanitary Care for Women in Indian Slums'. Journal of International Development. 29 (1): 32-51.

Garikipati, S., Docherty, R.J., Phillips-Howard, P.A., 2019. 'What's the bleeding problem? Policy and attitudes towards sustainable menstrual hygiene materials in India'. University of Liverpool Working Paper in Economics No. 201907.

Guterman, M., Mehta, P., Gibbs, M. 2007. 'Menstrual Taboos Among Major Religions'. The Internet Journal of World Health and Societal Politics. 5(2):1-7.

Hennegan, J., Dolan, C., Wu, M., Scott, L., Montgomery, P., 2016. Schoolgirls' experience and appraisal of menstrual absorbents in rural Uganda: a cross-sectional evaluation of reusable sanitary pads. Reprod. Health 13: 143. https://doi.org/10.1186/s12978-016-0260-7.

Jatan Sansthan, 2001. https://jatansansthan.org/uger-pads-2/ <Accessed on 22 April, 2020>.

Jayachandran, S., Jain, T., Dhar, D., 2018. 'Reshaping Adolescents' Gender Attitudes: Evidence from a SchoolBased Experiment in India'. Global Poverty Research Lab Working Paper No. 18-07.

Mahajan, T., 2019. 'Imperfect Information in Menstrual Health and the Role of Informed Choice'. Indian Journal of Gender Studies. 26(1\&2): 59-78.

Museum of Menstruation and Women's Health, 1988. http://www.mum.org/CuradsKotexads.htm $<$ Accessed on 20 April, 2020>.

Muralidharan, A., Patil, H., Patnaik, S. 2015. 'Unpacking the policy landscape for menstrual hygiene management: implications for school WASH programmes in India'. Waterlines. 34(1): 79-91.

National Family Health Survey- India. 2015-16. International Institute for Population Sciences. http://rchiips.org/NFHS/factsheet_NFHS-4.shtml <Accessed on 22 April, 2020>.

National MHM Guidelines. 2015. http://www.ccras.nic.in/sites/default/files/Notices/16042018 Menstrual_Hygiene Management.pdf $<$ accessed on 22 April, 2020>

National MHM Consultation. 2017. Menstrual hygiene products in India: The evolving landscape. Pushing the Boundaries on the MHM Dialogue in India. http://wateraidindia.in/wp- 
content/uploads/2017/06/MHMConsultation-All-briefs.pdf $<$ Accessed on 22 April, 2020>.

Peberdy, E., Jones, A., Green, D., 2019. 'A Study into Public Awareness of the Environmental Impact of Menstrual Products and Product Choice'. Sustainability. 11(2): 473-488.

Plan India Annual Report, 2012. Issuu. https://issuu.com/planindia/docs/eannual_report $<$ Accessed on 22 April, 2020>.

Sivakami, M., van Eijk, A.M., Thakur, H., Kakade, N., Patil, C., Shinde, S., Surani, N., Bauman, A., Zulaika, G., Kabir, Y., Dobhal, A., Singh, P., Tahiliani, B., Mason, L., Alexander, K., Thakkar, M., Laserson, K., Phillips-Howard, P. 2018. 'Effect of menstruation on girls and their schooling, and facilitators of menstrual hygiene management in schools: surveys in government schools in three states in India, 2015'. Journal of Global Health. 9(1): 010408.

Sommer, M., Vasquez, E., Worthington, N., Sahin, M., 2012. 'WASH in Schools Empowers Girls' Education: Proceedings of the Menstrual Hygiene Management in Schools Virtual Conference 2012'. United Nations Children's Fund and Columbia University, New York.

Sommer, M., Sahin, M., 2013. 'Overcoming the taboo: Advancing the global agenda for menstrual hygiene management for schoolgirls'. American Journal of Public Health. 103(9): 1556-1559.

The Logical Indian, 2019. 'Going Against The Trend: Instead Of Sanitary Pads, Kerala Municipality To Distribute 5,000 Menstrual Cups For Free'. https://thelogicalindian.com/news/projectthinkal/?infinitescroll $=1<$ Accessed on 22 April, 2020>

Thought Co. 2013. 'How long does it take garbage to decompose?' Environmental Issues, http://storage.neic.org/event/docs/1129/how long_does it take garbage to decompose.pdf $<$ Accessed on 22 April, 2020>

Torondel, B., Sinha, S., Mohanty, J.R., Swain, T., Sahoo, P., Panda, B., Nayak, A., Bara, M., Bilung, B., Cumming, O., Panigrahi, P., Das, P., 2018. 'Association between unhygienic menstrual management practices and prevalence of lower reproductive tract infections: a hospital-based cross-sectional study in Odisha, India'. National Centre for Biotechnology Information. 18(1): 473.

UN, 2019. World population prospects 2019. https://esa.un.org/unpd/wpp/<accessed 6.20.2019>

UNICEF. 2014. Proceedings of the MHM Virtual Conference.

van Eijk, A.M., Sivakami, M., Thakkar, M.B., Bauman, A., Laserson, K.F., Coates, S., Phillips-Howard, P.A., 2016. 'Menstrual hygiene management among adolescent girls in India: a systematic review and metaanalysis'. BMJ Open. 6: 010290.

van Eijk, A.M.V., Zulaika, G., Lenchner, M., Mason, L., Sivakami, M., Nyothach, E., Unger, H., Laserson, K., Philips-Howard, P.A., 2019. 'Menstrual cup use, leakage, acceptability, safety, and availability: a systematic review and meta-analysis'. The Lancet Public Health. 4(8): 376-393.

Venema, V., 2014. 'The Indian sanitary pad revolutionary'. BBC News. http://www.bbc.com/news/magazine$26260978<$ Accessed on 22 April, 2020>

Waheed, A., 2018. 'India's sanitary towel hero Pad Man bound for Bollywood glory'. The Guardian. https://www.theguardian.com/society/2018/feb/04/india-sanitary-towel-hero-padman-bollywood-gloryarunachalam-muruganantham $<$ Accessed on 22 April, 2020 $>$

WHO. 2010. Why urban health matters. <https://apps.who.int/iris/handle/10665/70230> (accessed 9.16.2019)

Wilmouth, R., Muller, N., Truyens, N., Hoppenjans, L., Sommer, M., Adelman, S., Hoffmann, V., 2013. 'Interactions between menstrual hygiene management and sanitation systems: Landscape analysis of menstrual hygiene products and a waste-loading model'. Path.

https://pdfs.semanticscholar.org/a737/78b61bcdfd695a1b37deae08ccf53dd06ce8.pdf (Accessed on 22 April, 2020).

World Population Datasheet, 2017. https://www.prb.org/wp-content/uploads/2017/08/WPDS-2017.pdf $<$ Accessed on 22 April, 2020>. 